'Centro de Geriatría y Gerontología, Depto. Medicina Interna, Facultad de Medicina, Pontificia Universidad Católica de Chile.

Residente de Geriatría Pontificia Universidad Católica de Chile. 2Psicóloga.

Recibido el 30 de marzo de 2010, aceptado el $26 \mathrm{de}$ abril de 2011

Correspondencia: a Dra. Paola Fuentes R. Residente de Geriatría, Centro de Geriatría PUC. Marcoleta 350, Santiago, Chile.

Fax 02-3546820 E-mail:paolafuentesr@ gmail.com

\section{Deterioro cognitivo e hiperparatiroidismo primario: resultado favorable después de cirugía. Caso clínico}

\author{
PAOLA FUENTES R. ${ }^{a}$, HOMERO GAC ${ }^{1}$, MARCELA CARRASCO ${ }^{1}$, \\ LORENA AGUILAR ${ }^{1,2, b}$, PEDRO PAULO MARÍN. ${ }^{1}$
}

\section{Cognitive impairment associated to primary hyperparathyroidism. Report of one case}

We report a 79-year-old male patient presenting with progressive memory loss associated with anxiety and muscular pain. An extensive biochemical control revealed high serum calcium and parathormone levels, and normal thyroid hormones. Cognitive assessment prior to surgery was compatible with mild cognitive impairment, showing significant improvement two months after parathyroidectomy. Our case suggests that, although rare, primary hyperparathyroidism should be considered as a possible cause of cognitive decline in the elderly.

(Rev Med Chile 2011; 139: 638-641).

Key words: Aged; Dementia; Hyperparathyroidism.
E l envejecimiento de la población es un éxito de la salud pública pero trae nuevos desafíos médicos y sociales ${ }^{1,2}$. La Geriatría ha recalcado la importancia de mantener la funcionalidad (física y mental) a toda edad, por lo que en clínica siempre debemos buscar causas corregibles para tratarlas a tiempo y no denegar tratamientos sólo por la edad del paciente ${ }^{3,4}$. El deterioro cognitivo es una de las entidades que aumentan con la edad por lo que siempre se debe evaluar, ya que hay varias causas descritas como corregibles ${ }^{5}$ y una es el hiperparatiroidismo primario que, aunque infrecuente, debemos tenerlo presente si encontramos hipercalcemia en el paciente mayor ${ }^{6-9}$.

Presentamos el caso de un sujeto de 79 años, con hiperparatiroidismo primario comprobado, que se presentó a la consulta de Geriatría para estudio de deterioro cognitivo.

\section{Caso clínico}

Paciente hombre de 79 años, casado, microempresario, con nivel educacional universitario, en tratamiento con escitalopram $10 \mathrm{mg}$ al día por un síndrome depresivo. Consultó por cuadro de cinco meses de evolución caracterizado por ansiedad, dolores musculares generales que eran mayores en la región del cuello, siendo lo principal una pérdida progresiva de su memoria, lo cual fue confirmado también por sus familiares directos. El examen físico fue normal. Se le solicitó TAC cerebral que mostró signos de pérdida de volumen encefálico, descritos como acordes a la edad. El estudio de laboratorio que incluyó hemograma, VHS, función renal, hepática y hormonas tiroideas fue normal, salvo hipercalcemia $(10,7 \mathrm{mg} / \mathrm{dL})$, con fósforo de $2,5 \mathrm{mg} / \mathrm{dL}$, albúmina de 4,3 g/dL. Esto motivó la solicitud de PTH, la que resultó ser de 106,5 pg/ $\mathrm{ml}$ (referencia hasta $65 \mathrm{pg} / \mathrm{ml}$ ). La vitamina D3 en plasma era $22,5 \mathrm{ng} / \mathrm{ml}$. Fue hospitalizado para estudio, solicitándose cintigrama paratiroideo con MIBI planar y SPECT que demostró una glándula paratiroides inferior izquierda hiperfuncionante. La densitometría ósea reveló osteoporosis a nivel de columna lumbar (T-score de $-3,3$ ), cadera derecha (T-score de $-3,5)$ y cadera izquierda (T-score de $-3,3)$. La calciuria en orina de 24 horas fue de 390 mg/24 horas. Cintigrama óseo mostró leve 

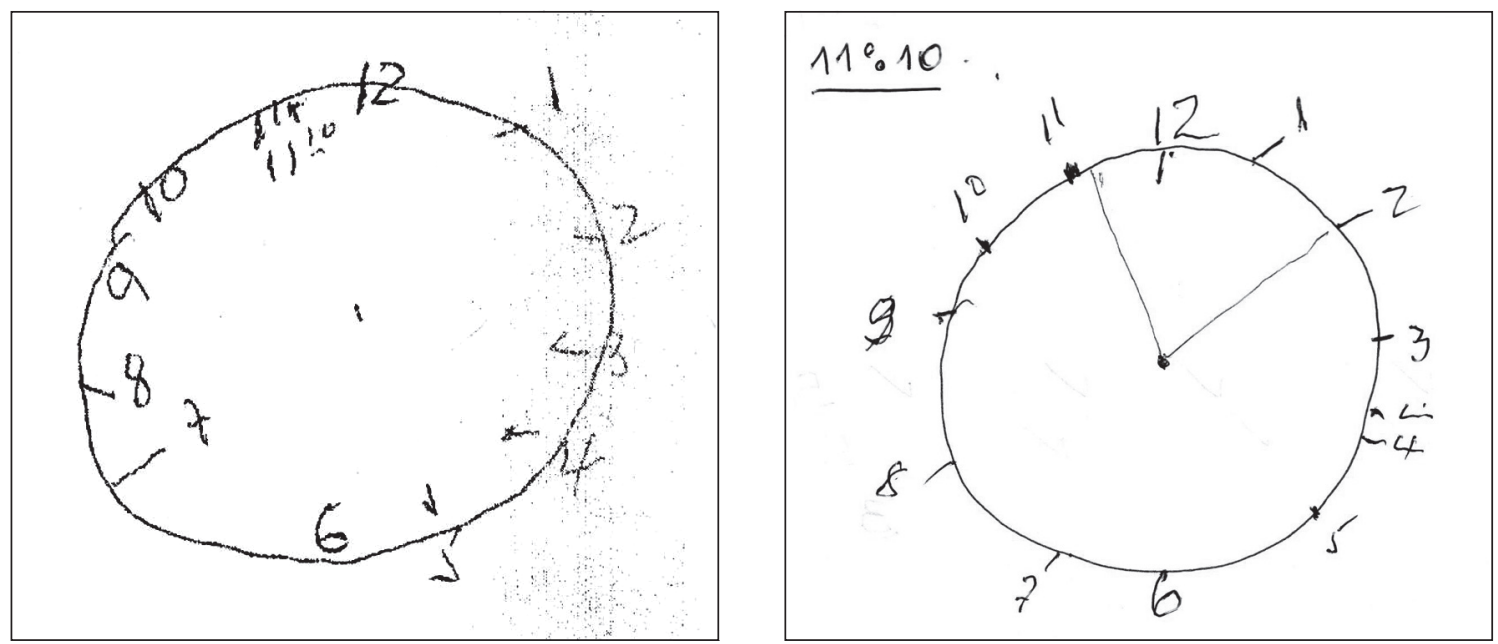

Figura 1. Test del Reloj previo a cirugía (izquierda) y 2 meses posterior a cirugía (derecha).

Tabla 1. Comparación tests cognitivos previo a cirugía y $\mathbf{2}$ meses después

\begin{tabular}{|lcc|}
\hline Test & $\begin{array}{c}\text { Previo a } \\
\text { cirugía }\end{array}$ & $\begin{array}{c}\text { 2 meses } \\
\text { post cirugía }\end{array}$ \\
\hline MMSE & 20 & 26 \\
Test del reloj & $1 / 4$ & $3 / 4$ \\
$\begin{array}{l}\text { Lista de animales en } \\
1 \text { minuto }\end{array}$ & 6 & 9 \\
\hline
\end{tabular}

aumento de captación en aspecto posterior de L5$\mathrm{S} 1$ y en rótula derecha, compatible con cambios de tipo degenerativo. Sin evidencias de compromiso óseo secundario.

Durante su hospitalización se realizó valoración geriátrica integral, demostrándose que era independiente en sus actividades básicas e instrumentales de la vida diaria. El paciente se encontraba vigil, orientado témporo-espacialmente, atento, capaz de invertir series (días de la semana) sin dificultad. Puntaje de Mini Mental Test de Folstein (MMSE) 20/30, con fallas en memoria, resta de series y copia de dibujo. Capacidad de abstracción conservada, con lenguaje coherente y pensamiento organizado. Lista de animales: 6 en 1 minuto y test del reloj no logrado (puntaje 1/4), con fallos en orientación y organización espacial y compromiso ejecutivo (Figura 1). Esta valora- ción cognitiva es compatible con los criterios de deterioro cognitivo leve, ya que el paciente no presentaba interferencia funcional por su deterioro cognitivo (Mild cognitive impairment) ${ }^{10}$.

Se decidió en forma interdisciplinaria y en conjunto con paciente y familia realizar paratiroidectomía inferior izquierda, evolucionando sin complicaciones y dado de alta al tercer día post operado. La biopsia demostró un adenoma paratiroideo oxofílico de 1,3 g.

$\mathrm{Al}$ mes del alta se controlaron exámenes: calcemia de 9,5 mg/dL, fósforo de $3 \mathrm{mg} / \mathrm{dL}, \mathrm{PTH} 45,5$ $\mathrm{pg} / \mathrm{ml}$ (normal), albúmina de 4,2 g/dL; estando el paciente sin dolores musculares, volvió a su trabajo regular y refiriendo que su estado cognitivo estaba mejorando.

A los dos meses, se controló en policlínico de Geriatría, realizándose valoración cognitiva. El MMSE fue 26/30 (previo de 20), fallando sólo en resta de series. Lista de animales subió a 9 en un minuto. Logró el test del reloj, observándose mejor organización espacial y menores fallas ejecutivas (Puntaje 3 de 4) (Tabla 1, Figura 1).

\section{Discusión}

Con el envejecimiento de la población, las demencias se vuelven un problema de salud pública creciente.

Una vez diagnosticada una demencia, el si- 
guiente paso es determinar su etiología, considerando la presencia de demencias potencialmente reversibles o tratables. Se considera una demencia reversible a aquellos cuadros de deterioro cognitivo ocasionados por un factor identificable cuyo tratamiento en un plazo razonable de tiempo permite la mejoría del proceso o al menos frena la progresión. Se prefiere el término demencia tratable, ya que no todas tienen reversibilidad total. Se considera actualmente que sólo existe $5-10 \%$ de demencias tratables. Dentro de ellas encontramos causas neurológicas como lesiones estructurales (hematoma subdural, neoplasias cerebrales, hidrocefalia normotensiva), nutricionales (déficit de vitamina B12), infecciosas (abscesos cerebrales, neurosífilis), causas psiquiátricas (depresión, esquizofrenia) y causas endocrinas (hipotiroidismo, hipertiroidismo, hiperparatiroidismo $)^{5}$.

Teniendo esto en consideración, se solicitó a este paciente los exámenes generales recomendados para el estudio de deterioro cognitivo, encontrándose alteración en la calcemia y posteriormente de la PTH, llegándose al diagnóstico de hiperparatiroidismo primario.

El hiperparatiroidismo primario afecta con mayor frecuencia a mujeres post menopáusicas, produciéndose una alteración del calcio, fósforo y metabolismo óseo debido a secreción incrementada de PTH, manifestándose con hipercalcemia e hipofosfemia ${ }^{6,7}$. La mayoría son clínicamente asintomáticos. Pueden presentar compromiso renal (depósitos de calcio en el parénquima renal o nefrolitiasis recurrente), sistema esquelético (osteitis fibrosa quística) y tracto digestivo (úlcera péptica y pancreatitis). También se reportan alteraciones del sistema nervioso central, nervios periféricos (neuropatía periférica) y músculos ${ }^{12}$. Los síntomas neuropsiquiátricos incluyen: depresión en aproximadamente $66 \%$ de los casos, alteraciones de memoria en $25 \%$, psicosis y agitación en $10 \%$, confusión y cambios de personalidad ${ }^{8}$.

Este paciente mayor se presentó con un deterioro cognitivo progresivo de algunos meses de evolución que revirtió parcialmente al tratar quirúrgicamente el hiperparatiroidismo. El mecanismo por el cual se produce deterioro cognitivo en pacientes con hiperparatiroidismo primario es desconocido. El metabolismo del calcio puede tener influencia en el funcionamiento cortical superior $^{13}$. Hipercalcemias más severas pueden producir confusión, psicosis, coma e incluso muer- te. Se ha encontrado en los pacientes con hiperparatiroidismo primario niveles incrementados de calcio total e iónico en líquido cefalorraquídeo que se cree afectaría la función cognitiva ${ }^{14}$.

En general, el hiperparatiroidismo primario asintomático se maneja en forma médica ${ }^{12}$. Las alteraciones neurocognitivas son parte integral del diagnóstico del hiperparatiroidismo primario, pero no deben considerarse como indicación única para cirugía ${ }^{12}$. Los estudios sobre los efectos de la paratiroidectomía en la función cognitiva son controvertidos. Algunos estudios muestran mejoría de estos síntomas y mejor calidad de vida ${ }^{15-17}$.

Este caso nos sugiere que el hiperparatiroidismo primario puede ser una causa subestimada de deterioro cognitivo corregible.

\section{Referencias}

1. Marín PP. La situación del adulto mayor en Chile. Rev Med Chile 1997; 125: 1207-12.

2. Marín PP. Reflexiones para considerar en una política pública de salud para las personas mayores. Rev Med Chile 2007; 135: 392-98.

3. Marín PP. Reseña histórica geronto-geriátrica. En: Geriatría y Gerontología -tercera edición ampliada. Pedro Paulo Marín, H Gac, M Carrasco, editores. Santiago, Chile: Ediciones Universidad Católica; 2006. p. 15-8.

4. Los desafíos actuales del adulto mayor: cambiando la visión negativa de la tercera edad. Editado por Facultad de Medicina PUC, CIEDES y Colmena Golden Cross. Santiago: 2005.

5. Piccini C, Bracco L, Amaducci L. Treatable and reversible dementias: an update. Journal of the Neurological Sciences 1998; 172-81.

6. Marx SJ. Hyperparathyroid and Hypoparathyroid Disorders. N Engl J Med 2000; 343: 1863.

7. Aw D, Sahota O. Primary hyperparathyroidism in the older patient. CME Geriatric Medicine 2010; 12: 115-22.

8. Molaschi M, Ponzetto M, Romin R. Neuropsychologic symptoms of primary hyperparathyroidism in the elderly. Report of a clinical case. Minerva Endocrinologica 1994; 19: 169-74.

9. Joborn C, Hetta J, Frisk P, Palmer M, Akerstrom G, Ljunghall S. Primary hyperparathyroidism in patients with organic brain syndrome. Acta Medica Scandinavia 1986; 219: 91-8.

10. Papageorgiou SG, Christou Y, Kontaxis T, Bonakis A, Anagnostouli M, Potagas C, et al. Dementia as presen- 
ting symptom of primary hyperparathyroidism: favourable outcome after surgery. Clin Neurol Neurosurg 2008; 110: 1038-40.

11. Gauthier S, Reisberg B, Zaudig M, Petersen R, Ritchie K, Broich K, et al. Mild cognitive impairment. Lancet 2006; 367: 1262-70.

12. Fraser W. Seminar: Hyperparathyroidism. The Lancet 2009, 374: 145-178.

13. Roman SA, Sosa JA, Mayes L, Bouduourakis L, Lin R, Snyder PJ, et al. Parathyroidectomy improves neurocognitive deficit in patients with primary hyperparathyroidism. Surgery 2005; 138: 1121-9.

14. Cermik TF, Kaya M, Ugur-Altun B, Bedel D, Berkarda S, Yigitbasi O. Regional cerebral blood flow abnormalities in patients with primary hyperparathyroidism. Neuro- radiology 2007; 49: 379-85.

15. Prager G, Kalashek A, Kaczirek K, Passler C, Scheuba C, Sonneck G, et al. Parathyroidectomy improved cognition and retentiveness in patients with primary hyperparathyroidism. Surgery 2002; 132: 930-6.

16. Dotzenrath Cm, Kaetsch Ak, Pfingsten H, Cupisti K, Weyerbrock, Vossough A, et al. Neuropsychiatric and cognitive changes after surgery for primary hyperparathyroidism. World Journal of Surgery 2006; 30: 680-5.

17. Chiang CY, Andrewest DG, Anderson D, Devere M, Schweitzer, Zajac J. A controlled, prospective study of neuropsychological outcomes post parathyroidectomy in primary hyperparathyroid patients. Clinical Endocrinology 2005; 62: 99-104. 